\title{
Time-resolved fluoroimmunoassay for quantitative determination of tylosin and tilmicosin in edible animal tissues
}

\author{
WEI Shu ${ }^{1}$, LE Tao $^{1,2^{*}}$, CHEN Yong $^{2}$, XU Jian ${ }^{3}$, HE HongQiu ${ }^{2}$, NIU XiaoDong ${ }^{2} \&$ LUO JinHua ${ }^{2 *}$ \\ ${ }^{1}$ College of Life Science, Chongqing Normal University, Chongqing 401331, China; \\ ${ }^{2}$ Chongqing Academy of Science and Technology, Chongqing 401123, China; \\ ${ }^{3}$ Chongqing RuoFeng Bio-Tech Co., Ltd, Chongqing 401331, China
}

Received October 26, 2012; accepted January 23, 2013; published online February 28, 2013

\begin{abstract}
To quantitatively determine tylosin and tilmicosin in edible animal tissues, a time-resolved fluoroimmunoassay (TRFIA) has been developed and validated. For this purpose, desmycosin- $O$-carboxymethoxylamine-BSA was fixed onto microtiter plates, standards and samples were loaded and, finally, diluted europium-labeled anti-tylosin antibodies were added. Results show that the limit of detection for tylosin was $0.03 \mathrm{ng} \mathrm{mL}^{-1}$ and that for tilmicosin was $0.05 \mathrm{ng} \mathrm{mL}^{-1}$. The recoveries were $73.6 \%$ to $120.5 \%$, with coefficients of variation below $15.6 \%$ in various biological matrices spiked with tylosin and tilmicosin at concentrations of 50-200 $\mathrm{ng} \mathrm{g}^{-1}$. There was good correlation $\left(R^{2}>0.99\right)$ between the TRFIA, an enzyme-linked immunosorbent assay and high performance liquid chromatography data. In conclusion, the new TRFIA is applicable to the detection of tylosin and tilmicosin and is an effective and economical method that will enable high-throughput sample screening. The method is expected to be widely applicable.
\end{abstract}

tylosin, tilmicosin, monoclonal antibody, time-resolved fluoroimmunoassay, edible tissues

Citation: Wei S, Le T, Chen Y, et al. Time-resolved fluoroimmunoassay for quantitative determination of tylosin and tilmicosin in edible animal tissues. Chin Sci Bull, 2013, 58: 1838-1842, doi: 10.1007/s11434-013-5749-7

Tylosin, a macrolide antibiotic, is extensively applied in veterinary medicine because it is strongly resistant to most of Gram-positive and -negative bacteria, as well as Mycoplasma spp. and Chlamydia spp. [1]. Tilmicosin, (20-deoxo20-(3,5-dimethyl-piperidin-1-yl)-desmycosin, is a semisynthetic macrolide antibiotic with a spectrum of microbiological activity against Pasteurella spp., Mycoplasma spp., and a variety of Gram-positive organisms [2]. For these reasons, tylosin and tilmicosin are widely employed to treat a broad range of infections in domestic animals, especially respiratory diseases. The residues of these antibiotics may stay in the edible tissue of animals when misused, which may trigger allergies and select for resistance. Therefore, the use of tylosin and tilmicosin in feed additives was banned in the European Union in 1999 [3]. Within China, maximum residue limits (MRLs) have also been established for tylosin and tilmicosin. Therefore, there is a need to develop a sim-

*Corresponding authors (email: hnxylt@163.com; cqbio@126.com) ple and efficient detection method to screen tylosin and tilmicosin antibiotics in food producing animals.

For animal product safety control, several detection methods for tylosin and tilmicosin residues have been developed in recent years, including high performance liquid chromatography (HPLC) [4-8], thin-layer chromatography [9], HPLC coupled with mass spectrometry [10-15], and microbiological assays $[4,16]$. However, expensive equipment, skilled technicians and complicated sample pretreatments are required for those methods, making them unsuitable for high throughput field detection or routine screening. The microbiological assay is lengthy and insufficiently specific for analytical purposes. Immunoassays are valuable as rapid, low cost and high throughput tests for rapid screening of a large number of samples. A number of researchers have established enzyme-linked immunosorbent assays (ELISAs) for tylosin and tilmicosin [1,17-20]. ELISA is used frequently because it is highly sensitive, portable and suitable for large-scale use but it is imprecise and positively biased 
because of matrix effects [21,22].

A highly sensitive, dual-label, time-resolved fluoroimmunoassay (TRFIA) that can simultaneously detect tylosin and tilmicosin is described in this paper, which offers advantages of cost and time efficiency, allowing simplified assay protocols and small sample volumes [22]. Because of the unique fluorescent properties of lanthanide chelates, which have the advantages of high quantum yields, narrowband emission peaks, long Stoke's shifts and fluorescence lifetimes, TRFIA is distinct from conventional fluorescent dyes. The half-life of background fluorescence is very short so the long duration of lanthanide chelate emission allows longer measurement, which distinguishes specific fluorescence from the background. Thus, the sensitivity of a TRFIA is higher than that of conventional methods, which lessens the susceptibility of the TRFIA to matrix interference [21,23].

This is the first report describing an $\mathrm{Eu}^{3+}$ fluorescent lanthanides-based TRFIA with high sensitivity and costefficiency for detection of tylosin and tilmicosin in edible tissues of food-producing animals. Comparisons are made between the TRFIA and conventional HPLC and ELISA methods. Finally, TRFIA was validated by analysis of swine muscle and liver samples.

\section{Materials and methods}

\subsection{Materials and reagents}

Tylosin, tilmicosin, desmycosin (DES), $O$-carboxymethoxylamine hemihydrochloride (CMO), spiramycin, erythromycin, kitasamycin, azithromycin, josamycin, oleandomycin, roxithromycin, avermectin, ivermectin, ovalbumin (OVA), and bovine serum albumin (BSA) were purchased from Sigma (St. Louis, MO, USA). $N^{\prime}$-[p-Isothiocyanatobenzyl]diethylene-triamine $N^{1}, N^{2}, N^{3}, N^{4}$-tetraacetate-Eu ${ }^{3+}\left(\right.$ DTTA-Eu ${ }^{3+}$ ) was from the Tianjin Radio-Medical Institute (Tianjin, China). Anti-tylosin monoclonal antibody (anti-tylosin MAb), DES-CMO-BSA and DES-CMO-OVA were obtained from Huazhong Agricultural University (Wuhan, China). A mouse monoclonal antibody isotyping kit was purchased from Southern Biotech Co. (Beijing, China). Sephadex 6B and G-50 were obtained from Pharmacia (Uppsala, Sweden). Buffers were prepared in our laboratory. All chemicals and reagents were of analytical grade or better, and the percentage concentrations are reported by weight unless otherwise specified.

\subsection{Labeling of MAb with europium chelate}

Europium-labeled MAbs (tracers) were prepared as described in the literature [24]. Briefly, $1 \mathrm{mg}$ anti-tylosin MAb and $1 \mathrm{mg}$ DTTA-Eu ${ }^{3+}$ were mixed in $50 \mathrm{mmol} \mathrm{L}^{-1}$ sodium carbonate buffer ( $\mathrm{pH}$ 9.6) in an amber bottle and then kept at $4^{\circ} \mathrm{C}$ with stirring for over $24 \mathrm{~h}$. The mixture was purified by a Sephadex 6B/G-50 column and $0.1 \%(\mathrm{w} / \mathrm{v}) \mathrm{BSA}$ was added to the collected MAb-DTTA-Eu ${ }^{3+}$. This mixture of MAb-DTTA-Eu ${ }^{3+}$ and BSA was ready for use and can be preserved for one year when stored at $-20^{\circ} \mathrm{C}$.

\subsection{Pre-treatment of samples}

Samples, such as porcine muscles, porcine liver, chicken muscle, chicken liver and fish, obtained at a local supermarket, were analyzed by HPLC for the tylosin and tilmicosin contents. The negative control was samples without tylosin and tilmicosin contamination, as identified by an HPLC test. The sample $(2 \pm 0.005) \mathrm{g}$ was homogenized, mixed with $4 \mathrm{~mL}$ ethyl acetate, followed by $2 \mathrm{~min}$ vortexing and then $10 \mathrm{~min}$ centrifugation at $2000 \mathrm{r} \mathrm{min}^{-1}$. The supernatant was gathered and $4 \mathrm{~mL}$ ethyl acetate was added to the pellet and the above treatment was repeated. The supernatant was evaporated in a water bath under a gentle nitrogen flow at $60^{\circ} \mathrm{C}$. The extractions were resuspended in $1 \mathrm{~mL}$ of $10 \mathrm{mmol} \mathrm{L}^{-1} \mathrm{PBS}(\mathrm{pH} \mathrm{7.4)}$ and mixed thoroughly before TRFIA and ELISA analysis. The samples were pretreated for HPLC analysis, as described previously [25].

\subsection{The competitive TRFIA}

The 96-well Maxisorp microtiter plates (Nunc, Roskilde, Denmark) were coated overnight with conjugates (DESCMO-BSA, $1 \mathrm{mg} \mathrm{L}^{-1}$ ) diluted in carbonate buffer $(50 \mathrm{mmol}$ $\mathrm{L}^{-1}, \mathrm{pH}$ 9.6), followed by blocking for $2 \mathrm{~h}$ at $37^{\circ} \mathrm{C}$ with a blocking buffer $(50 \mathrm{mmol} / \mathrm{L}$ Tris- $\mathrm{HCl}, \mathrm{pH} 8.0$, containing $0.9 \% \mathrm{NaCl}, 0.5 \% \mathrm{BSA}, 0.4 \%$ stabilizing reagent, and $0.04 \%$ $\mathrm{NaN}_{3}$ ). The plate was then washed three times with a washing buffer $\left(10 \mathrm{mmol} \mathrm{L}^{-1}\right.$ Tris- $\mathrm{HCl}, \mathrm{pH} 8.0$, containing $0.9 \%$ $\mathrm{NaCl}$ and $0.1 \%$ Tween 20). Subsequently, $50 \mu \mathrm{L}$ standard solutions or samples and $50 \mu \mathrm{L}$ europium-labeled MAbs diluted with assay buffer were added to the plates. After incubation at $37^{\circ} \mathrm{C}$ for $1 \mathrm{~h}$, the plates were washed six times, and an enhancement solution $\left(0.1 \mathrm{~mol} \mathrm{~L}^{-1}\right.$ potassium biphthalate-acetic acid buffer containing $15 \mu \mathrm{mol} \mathrm{L}{ }^{-1} \beta$-naphthoyltrifluoroacetone and $0.1 \%(\mathrm{w} / \mathrm{v})$ Triton $\mathrm{X}-100)$ was added to each well. The plates were then shaken for $5 \mathrm{~min}$ for reaction and then rested for $10 \mathrm{~min}$. The fluorescence was measured using a VICTOR ${ }^{2}$ multilabel counter (PerkinElmer Wallac, Turku, Finland) at excitation wavelength $340 \mathrm{~nm}$ and emission wavelength $615 \mathrm{~nm}$, with a delay time of $400 \mu \mathrm{s}$. The tylosin and tilmicosin contents in the samples were calculated from standard curves. The half-maximal inhibition concentration $\left(\mathrm{IC}_{50}\right)$ was determined as a measure of the sensitivity of the TRFIA.

\subsection{Comparison of the TRFIA with ELISA and HPLC}

To further demonstrate the capability and accuracy of the TRFIA, a comparison between TRFIA, ELISA and HPLC 
was conducted using samples (liver and muscle) from an animal-feeding experiment. Duroc castrated pigs with weights of 13-15 kg were kept separately in an animal facility for one week. Eight pigs were randomly divided into control and test groups. The control group $(n=1)$ was under mock intramuscular treatment with another drug. The test group $(n=3)$ was treated by tylosin intramuscularly at a dose of bodyweight (BW) $10 \mathrm{mg} \mathrm{kg}^{-1}$ daily for 5 continuous days. The animals were euthanized at days 0,1 , and 3 of withdrawal after tylosin treatments. The muscle and liver samples were collected separately and stored at $-20^{\circ} \mathrm{C}$ until analysis by TRFIA, ELISA and HPLC.

\section{Results and discussion}

\subsection{Preparation of optimum working solutions for antibodies}

Tylosin or tilmicosin in a series of dilutions were added to the coated plate. As shown in Figure 1, the anti-tylosin MAb solutions diluted at 1:4000-1:10000 produced inhibitory rates of $50 \%-30 \%$. The working concentration for the anti-tylosin MAb was 1:5000.

\subsection{Cross-reactivities of MAb}

The cross-reactivities between tilmicosin, tylosin, and their structural analogues (desmycosin, 5- $O$-mycaminosyltylonolide, spiramycin, erythromycin, kitasamycin, azithromycin, josamycin, oleandomycin, roxithromycin, avermectin, ivermectin) were tested to assess specificity. As shown in Table 1, the MAb showed cross-reactivity towards tylo$\sin (100 \%)$, tilmicosin $(51 \%)$, desmycosin $(176 \%)$, and 5-O-mycaminosyltylonolide (4.5\%), but did not generate significant cross-reactivity $(<0.1)$ with the other antibiotics listed above. Thus, the TRFIA could be employed as a screening method for multiresidue detection.

\subsection{The standard curve for the TRFIA}

The optimum conditions for TRFIA were a coating antigen

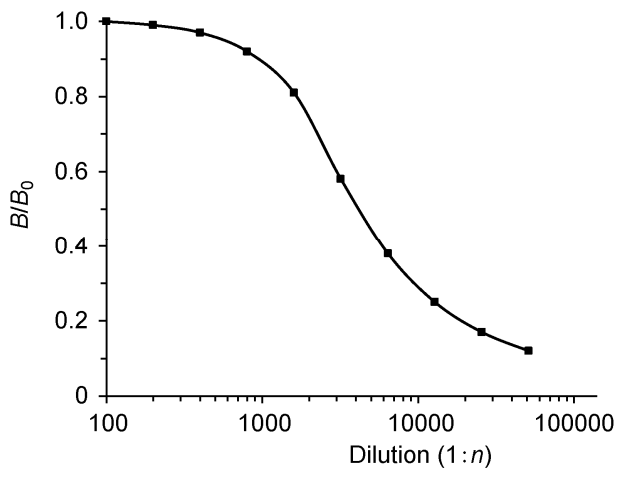

Figure 1 Dilution curve of MAb by TRFIA.
Table 1 Cross-reactivity of macrolide antibiotics by the TRFIA based on $\mathrm{MAb}$

\begin{tabular}{lcc}
\hline \multicolumn{1}{c}{ Competitor } & $\mathrm{IC}_{50}\left(\mathrm{ng} \mathrm{mL}^{-1}\right)$ & Cross-reactivity (\%) \\
\hline Tylosin & 0.98 & 100 \\
Tilmicosin & 1.92 & 51 \\
Desmycosin & 0.56 & 176 \\
5-O-mycaminosyltylonolide & 21.78 & 4.5 \\
Spiramycin & $>1000$ & $<0.1$ \\
Erythromycin & $>1000$ & $<0.1$ \\
Kitasamycin & $>1000$ & $<0.1$ \\
Azithromycin & $>1000$ & $<0.1$ \\
Josamycin & $>1000$ & $<0.1$ \\
Oleandomycin & $>1000$ & $<0.1$ \\
Roxithromycin & $>1000$ & $<0.1$ \\
Avermectin & $>1000$ & $<0.1$ \\
Ivermectin & $>1000$ & $<0.1$ \\
\hline
\end{tabular}

(DES-CMO-OVA) concentration of $1 \mathrm{mg} \mathrm{L}^{-1}$ and a MAb dilution ratio of $1: 8000$, which generated an optimal linear portion of the response curve. These conditions were adopted for subsequent experiments. The standard curves plotted by TRFIA analysis showed that the working concentration ranged from 0.01 to $100 \mathrm{ng} \mathrm{mL}^{-1}$ (Figure 2). Good linearity was obtained in the range of 0.08 to $25 \mathrm{ng} \mathrm{mL}^{-1}$, with acceptable correlation coefficients $\left(R^{2}=96.83 \%\right.$ $99.19 \%)$. With regard to assay sensitivity, $\mathrm{IC}_{50}$ values were calculated to be 0.89 and $1.73 \mathrm{ng} \mathrm{mL}^{-1}$ for tylosin and tilmicosin, respectively. The limit of detection (LOD) was expressed as mean $+3 \times$ standard deviations [22]. Blank samples were determined by repeated analysis $(n=20)$ of tylosin/tilmicosin-free samples. The LODs of the TRFIA for tylosin and tilmicosin were 0.03 and $0.05 \mathrm{ng} \mathrm{mL}^{-1}$, respectively. The definition for limit of quantification (LOQ) was the tylosin/tilmicosin concentration of samples with a $B / B_{0}$ ratio of approximately 0.8 and coefficient of variation $(\mathrm{CV})$ less than $20 \%$ [22]. The LOQ for tylosin was $0.07 \mathrm{ng} \mathrm{mL}^{-1}$, with CVs of 4.7, while the LOQ for tilmicosin was 0.11 $\mathrm{ng} \mathrm{mL} \mathrm{L}^{-1}$, with CVs of 5.3 .

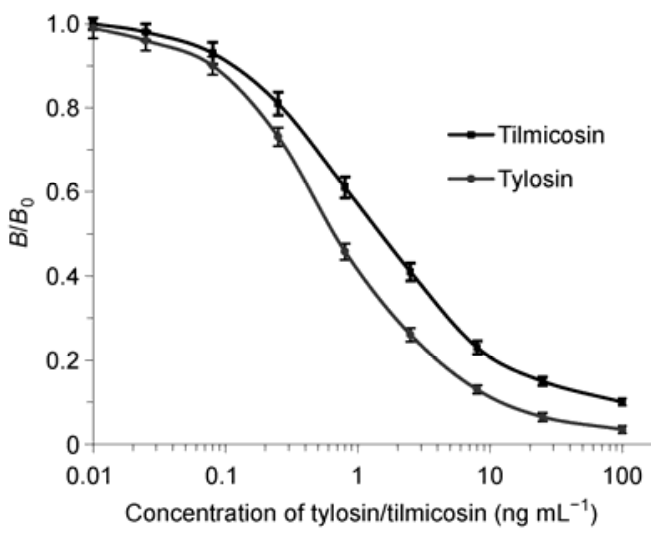

Figure 2 Calibration curve of tylosin/tilmicosin TRFIA. 


\subsection{Recovery and reliability of the TRFIA assay}

The MRL for tylosin or tilmicosin is $100 \mathrm{ng} \mathrm{g}^{-1}$ in muscle and liver and $50 \mathrm{ng} \mathrm{g}^{-1}$ in egg [1]. The recoveries and repeatability of various biological matrices spiked with tylosin or tilmicosin at levels of $0.5 \times$ MRL, $1 \times$ MRL, and $2 \times$ MRL are listed in Table 2. Close correlations were obtained between the measured values and the fortified concentrations at the three spiked levels. The recovery for tylosin ranged between $73.6 \%$ and $108.5 \%$ and that for tilmicosin ranged between $75.1 \%$ and $120.5 \%$. The repeatability of the assay was validated by calculating CVs, which were less than $15.6 \%$.

\subsection{Comparison between TRFIA, ELISA and HPLC}

Table 3 displays a summary of the results from TRFIA, HPLC and ELISA analyses, which reveals that 1-3 withdrawal days were needed for the attenuation of drug concentration to the MRLs levels (100 $\mathrm{ng} \mathrm{g}^{-1}$ ) in both swine muscle and liver. For quantitative analyses, TRFIA, ELISA and HPLC methods showed good correlation coeficients
$\left(R^{2}>0.99\right)$ in a side-by-side comparison. However, the TRFIA results are closer to HPLC results than to ELISA results for positive tylosin samples collected intraday and at the first day of withdrawal.

\section{Conclusions}

In this study, a simple and efficient TRFIA method has been established, which enables the analysis of tylosin and tilmicosin in edible animal tissues. The TRFIA method with europium tracer has a significantly increased sensitivity compared with the conventional ELISA. To validate the new method, comparisons were made between TRFIA, HPLC and ELISA in animal experiments, by which good correlations $\left(R^{2}>0.99\right)$ were obtained and the new method was validated. The detection limits, CVs, and recoveries satisfy the standards of the USA, EU, Canada, and China. Therefore, the new TRFIA described here is a rapid, accurate, and economical method for detecting tylosin, tilmicosin and their metabolites, simultaneously, in animal tissues.

Table 2 Recoveries of tylosin and tilmicosin spiked in tissues by the TRFIA $(n=15)^{\text {a) }}$

\begin{tabular}{|c|c|c|c|c|c|}
\hline \multirow{2}{*}{ Sample } & \multirow{2}{*}{ Spiked level $\left(\mathrm{ng} \mathrm{g}^{-1}\right)$} & \multicolumn{2}{|l|}{ Tylosin } & \multicolumn{2}{|c|}{ Tilmicosin } \\
\hline & & Mean recovery $\left(\mathrm{ng} \mathrm{g}^{-1}\right)$ & $\mathrm{CV}(\%)$ & Mean recovery $\left(\mathrm{ng} \mathrm{g}^{-1}\right)$ & $\mathrm{CV}(\%)$ \\
\hline \multirow[t]{3}{*}{ Porcine muscle } & 50 & 94.6 & 9.6 & 88.7 & 12.5 \\
\hline & 100 & 73.6 & 5.3 & 108.7 & 15.6 \\
\hline & 200 & 95.3 & 4.8 & 112.5 & 7.9 \\
\hline \multirow[t]{2}{*}{ Porcine liver } & 50 & 103.7 & 12.4 & 95.4 & 11.3 \\
\hline & 100 & 79.4 & 10.3 & 106.8 & 10.8 \\
\hline \multirow[t]{3}{*}{ Chicken } & 50 & 87.4 & 7.5 & 85.3 & 9.7 \\
\hline & 100 & 97.6 & 4.7 & 99.8 & 11.6 \\
\hline & 200 & 78.2 & 13.2 & 76.2 & 10.7 \\
\hline \multirow[t]{3}{*}{ Chicken liver } & 50 & 102.6 & 10.6 & 93.2 & 14.8 \\
\hline & 100 & 84.2 & 6.4 & 75.1 & 8.6 \\
\hline & 200 & 108.5 & 5.9 & 84.9 & 5.6 \\
\hline \multirow[t]{2}{*}{ Fish } & 25 & 96.3 & 9.7 & 100.8 & 12.8 \\
\hline & 100 & 88.3 & 10.5 & 96.3 & 13.5 \\
\hline
\end{tabular}

a) The test was repeated three times with five replicates per concentration.

Table 3 Result analyses of HPLC, ELISA and TRFIA, for the detection of tylosin in swine muscle and liver samples from animal experiments (mean \pm SD, $n=5)^{\text {a) }}$

\begin{tabular}{|c|c|c|c|c|}
\hline Withdrawals (d) & Samples & $\operatorname{HPLC}\left(\mathrm{ng} \mathrm{g}^{-1}\right)$ & ELISA $\left(\mathrm{ng} \mathrm{g}^{-1}\right)$ & TRFIA $\left(\right.$ ng g $\left.^{-1}\right)$ \\
\hline \multirow[t]{2}{*}{0} & Muscle & $186.4 \pm 13.8$ & $172.9 \pm 23.5$ & $185.2 \pm 9.3$ \\
\hline & Liver & $487.5 \pm 48.6$ & $448.3 \pm 50.4$ & $485.6 \pm 27.6$ \\
\hline \multirow[t]{2}{*}{1} & Muscle & $78.3 \pm 13.8$ & $67.5 \pm 4.2$ & $77.9 \pm 10.5$ \\
\hline & Liver & $136.2 \pm 15.2$ & $119.7 \pm 16.2$ & $132.2 \pm 10.6$ \\
\hline 3 & Muscle & $<50$ & $<20$ & $18.7 \pm 4.3$ \\
\hline
\end{tabular}

a) SD means of standard deviation. LOD of tylosin by HPLC method was $50 \mathrm{ng} \mathrm{g}^{-1}$. 
This work was supported by the Scientific and Technological Research Project of Chongqing (CSTC2011ggB10009, cstc2012pt-kyys10002, cstc2012cx-rkxB10002), the National Natural Science Foundation of China (81202438) and the Natural Science Foundation of the Chongqing Education Committee (KJ110605).

1 Peng D P, Ye S Q, Wang Y L, et al. Development and validation of an indirect competitive enzyme-linked immunosorbent assay for the screening of tylosin and tilmicosin in muscle, liver, milk, honey and eggs. J Agric Food Chem, 2012, 60: 44-51

2 Ose E. In vitro antibacterial properties of EL-870, a new semi-synthetic macrolide antibiotic. J Antibiot, 1987, 40: 190

3 Council Regulation (EC) No 2821/98 of 17 December 1998 amending, as regards withdrawal of the authorisation of certain antibiotics, Directive 70/524/EEC concerning additives in feedingstuffs. Off $\mathrm{J}$ Eur Communities, 1998, L351: 4-5

4 Litterio N J, Calvinho L F, Flores M M, et al. Microbiological screening test validation for detection of tylosin excretion in milk of cows with low and high somatic cell counts. J Vet Med A, 2007, 54: 30-35

5 Juhel-Gaugain M, Anger B, Laurentie M. Multiresidue chromatographic method for the determination of macrolide residues in muscle by high-performance liquid chromatography with UV detection. J AOAC Int, 1999, 82: 1046-1053

6 Stobba-Wiley C M, Chang J P, Elsbury D T, et al. Determination of tilmicosin residues in chicken, cattle, swine, and sheep tissues by liquid chromatography. J AOAC Int, 2000, 83: 837-846

7 Stobba-Wiley C M, Readnour R S. Determination of tilmicosin residues in cow and sheep milk by liquid chromatography. J AOAC Int, 2000, 83: 555-562

8 Clark C R, Dowling P M, Boison J O. Development and validation of a method for determination of tilmicosin residues in equine plasma and tissues using hplc. J Liq Chromatogr Relat Technol, 2009, 32: 2839-2856

9 Vincent U, Gizzi G, von Holst C, et al. Validation of an analytical method for the determination of spiramycin, virginiamycin and tylosin in feeding-stuffs by thin-layer chromatography and bio-autography. Food Addit Contam A, 2007, 24: 351-359

10 Cherlet M, De Baere S, Croubels S, et al. Quantitation of tylosin in swine tissues by liquid chromatography combined with electrospray ionization mass spectrometry. Anal Chim Acta, 2002, 473: 167-175

11 Horie M, Takegami H, Toya K, et al. Determination of macrolide antibiotics in meat and fish by liquid chromatography-electrospray mass spectrometry. Anal Chim Acta, 2003, 492: 187-197

12 Thompson T S, Noot D K, Calvert J, et al. Determination of lincomycin and tylosin residues in honey using solid-phase extraction and liquid chromatography-atmospheric pressure chemical ionization mass spectrometry. J Chromatogr A, 2003, 1020: 241-250

13 Benetti C, Dainese N, Biancotto G, et al. Unauthorised antibiotic treatments in beekeeping: Development and validation of a method to quantify and confirm tylosin residues in honey using liquid chromatography-tandem mass spectrometric detection. Anal Chim Acta, 2004, 520: 87-92

14 Juan C, Moltó J C, Manes J, et al. Determination of macrolide and lincosamide antibiotics by pressurised liquid extraction and liquid chromatography-tandem mass spectrometry in meat and milk. Food Control, 2010, 21: 1703-1709

15 Bogialli S, Ciampanella C, Curini R, et al. Development and validation of a rapid assay based on liquid chromatography-tandem mass spectromtetry for determining macrolide antibiotic residues in eggs. $\mathrm{J}$ Chromatogr A, 2009, 1216: 6810-6815

16 Pol-Hofstad I, Driessen-Van Lankveld W, Tomassen M, et al. Collaborative study of a microbiological screening method (three-plate) for the banned antimicrobial growth promotors tylosin, virginiamycin, spiramycin, zinc bacitracin and avoparcin in animal feed. Food Addit Contam A, 2008, 25: 1465-1474

17 Jackman R, Spencer Y, Silverlight J, et al. Development of antibodies to tilmicosin and their use in the immunolocalization of the antibiotic in porcine lung tissue. J Vet Pharmacol Ther, 1997, 20: 131-132

18 Silverlight J, Brown A, Jackman R. Antisera to tilmicosin for use in ELISA and for immunohistochemistry. Food Agric Immunol, 1999, 11: 321-328

19 Beier R C, Creemer L C, Ziprin R L, et al. Production and characterization of monoclonal antibodies against the antibiotic tilmicosin. $\mathrm{J}$ Agric Food Chem, 2005, 53: 9679-9688

20 Situ C, Elliott C T. Simultaneous and rapid detection of five banned antibiotic growth promoters by immunoassay. Anal Chim Acta, 2005, 529: 89-96

21 Shen J Z, Zhang Z, Yao Y, et al. A monoclonal antibody-based time-resolved fluoroimmunoassay for chloramphenicol in shrimp and chicken muscle. Anal Chim Acta, 2006, 575: 262-266

22 Zhang J, Gao L, Zhou B, et al. Simultaneous detection of deoxynivalenol and zearalenone by dual-label time-resolved fluorescence immunoassay. J Sci Food Agric, 2011, 91: 193-197

23 Zhang Z, Liu J F, Yao Y, et al. A competitive dual-labelled time-resolved fluoroimmunoassay for simultaneous determination of chloramphenicol and ractopamine in swine tissues. Chin Sci Bull, 2011, 56: 1543-1547

24 Zhang Z, Liu J F, Shao B, et al. Time-resolved fluoroimmunoassay as an advantageous approach for highly efficient determination of sulfonamides in environmental waters. Environ Sci Technol, 2009, 44: 1030-1035

25 Prats C, Francesch R, Arboix M, et al. Determination of tylosin residues in different animal tissues by high performance liquid chromatography. J Chromatogr B, 2002, 766: 57-65

Open Access This article is distributed under the terms of the Creative Commons Attribution License which permits any use, distribution, and reproduction in any medium, provided the original author(s) and source are credited. 